\title{
Listening to Everyone's Voice Enhances Learning $(\mathrm{LEVEL})^{*}$
}

\author{
Kerri Stone \\ Department of Mathematical and \\ Computer Sciences \\ Colorado School of Mines \\ Golden, CO 80401 \\ Email: kstone@mines.edu
}

\author{
Doug Hakkarinen \\ Department of Mathematical and \\ Computer Sciences \\ Colorado School of Mines \\ Golden, CO 80401 \\ Email: dhakkari@mines.edu
}

\author{
Tracy Camp \\ Department of Mathematical and \\ Computer Sciences \\ Colorado School of Mines \\ Golden, CO 80401 \\ Email: tcamp@mines.edu
}

\begin{abstract}
Participation in elementary school classroom settings is dependent on students' abilities to process questions quickly. Pedagogical studies theorize that some students are able to answer a question before other students are able to process the question, creating a division in classroom participation. This paper presents LEVEL (Listening to Everyone's Voice Enhances Learning), a wireless sensor network application to balance, diversify, and encourage in-class participation. LEVEL offers two operating modes: a centralized interface and a distributed adaptive personal interface. The centralized interface features a traffic light to compel students to wait before responding to a question; we LEVEL the participation in the class by visually informing students when a question can be answered. The distributed interface adaptively sets a participation time delay on a per student basis predicated upon previous participation. LEVEL brings a wireless sensor network application to primary school classrooms. LEVEL connects student learning with technology while providing an enhanced in-class experience for all students.
\end{abstract}

\section{INTRODUCTION}

One issue in primary education is the verification of student learning through participation. Student participation frequently takes the form of audible responses to questions from the teacher. Commonly, the teacher asks a question, waits for students to indicate willingness to respond by raising a hand, and calls upon a student to respond. This format has some limitations. The distribution of students within a class who participate is generally not uniform. Certain students raise their hands quicker and thus answer more questions than their peers. This behavior reduces opportunities for other students to participate, impairing their learning. Overall, it would be beneficial to diversify the students that answer questions.

Some common options to address participation concerns include exploring alternative methods of seeking responses [1], [2]. For example, teachers may select students that do not participate as much, or call on students who do not have their hand raised. As an alternative, we propose a method that allows voluntary self-selection while increasing the fraction of students participating. [3], [4], and [5] theorize that students formulate answers only until they see another student's hand raised. Under this theory, student participation rates could be improved if students who raise their hands quickly would delay

\footnotetext{
${ }^{*}$ This work supported in part by NSF Grant CNS-0940632.
}

raising their hands. In this way, the majority of students have sufficient time to internalize the question before the 'quick' students raise their hands. This paper presents a system that tells students when it is acceptable to raise their hands.

We present LEVEL (Listening to Everyone's Voice Enhances Learning), a wireless sensor network to balance participation in the classroom. To meet our goal, we need to determine when a teacher presents a question. A good indication that it is time to answer a question is when the teacher has ceased talking, as often this will indicate that the teacher is soliciting responses. Many other possible interfaces exist. For example, the teacher could actively indicate that a response is requested by pressing a button or through a software interface. It is preferable, however, if the teacher can focus on the course material rather than on an unnatural interface.

LEVEL must also easily inform the students when it is appropriate to raise a hand to respond to a question. Thus, a widely accessible symbol is desired. For example, the symbols of a red light (stop) and a green light (go) are pervasive in society. LEVEL utilizes these light colors to communicate that it is acceptable to raise a hand when the light is green, and it is not acceptable to raise a hand when the light is red.

\section{RELATED WORK}

The benefits of increasing the wait time before students answer questions in the classroom setting is well documented [3], [4], [5]. Increased wait times result in a diversification of in-class participation, which has the positive outcome of an enhanced classroom learning experience for students [5]. Two methods to increase wait time are introduced in [2]. The two methods include (1) training teachers to wait longer for responses, and (2) using a small electronic device to measure student and teacher pause times and, subsequently, informing teachers of their wait times. Both methods to increase wait times in [2] require teacher training. LEVEL implements a wireless sensor network (WSN) system to autonomously compel students to wait before raising hands in the classroom.

EduClick is a popular system used in education to diversify participation [1]. EduClick implements a visual interface to display student answers. Students express their answers through a 'clicker' hand-held device. EduClick helps encour- 
age participation among students; however, EduClick requires that questions be phrased in a multiple choice or true/false manner. Furthermore, EduClick does not employ a wait time before students are allowed to answer with the 'clicker'.

Traffic lights have been used in classrooms to help monitor ambient noise levels and provide visual feedback to students and teachers [6], [7]. These devices activate lights based on the sensed noise in the classroom. Traffic lights are used to reduce the overall noise level in the room rather than facilitate classroom participation. Prior use of traffic lights in the classroom setting indicate that the traffic light symbol is effective in communicating feedback to students.

\section{DESIGN AND IMPLEMENTATION}

LEVEL has two operating modes: centralized and distributed. In the centralized configuration, an acoustic sensor detects when the teacher is speaking and a traffic light is used to signal student participation. The centralized application is called LEVEL-TrafficLight (LEVEL-TL). The distributed version of LEVEL employs an acoustic sensor for each student and offers students individual feedback on their historical inclass participation. The distributed version of LEVEL is called LEVEL-InteractiveStudent (LEVEL-IS). For a detailed report on LEVEL-TL and LEVEL-IS, please see [8]. (LEVEL source code is available at http://github.com/kstone/LEVEL.)

Both LEVEL-TL and LEVEL-IS utilize a teacher mote implemented as a TelosB [9] equipped with an EasySen Multi-Modality Sensor Board [10]. Upon boot, the teacher mote calibrates its acoustic sensor. This calibration period is used to acquire a baseline classroom noise level. During this calibration period the noise level in the classroom should be representative of a 'quiet' classroom. The teacher mote uses the calibrated baseline acoustic value to implement a semisupervised anomaly detection algorithm to determine when the teacher is speaking.

\section{A. Anomaly Detection}

We performed an empirical study to determine the distribution of acoustic readings at different noise levels. For each noise level condition, the raw output from the sensor was recorded for a period of 150 seconds at $1 \mathrm{~Hz}$. The first noise level studied was ambient noise, referred to as the baseline acoustic level, where we did not talk. The acoustic noise level when a person is talking, referred to as talking acoustic level, was then studied. In this case, one person read a passage of text for the duration of the test. The distribution of these two studies showed that the talking acoustic level has a higher variance in acoustic readings than the baseline acoustic level. This result indicates that it is possible to differentiate between 'talking' and 'not talking' using the standard deviation of the acoustic noise levels.

We then examined the false-negative $(F N)$ rate, or the probability of not detecting the teacher talking, when the teacher is talking. We analyzed the distribution of the talking acoustic level samples with respect to the mean and standard deviation of the baseline acoustic level. Each talking acoustic level sample had a probability of approximately 0.325 of

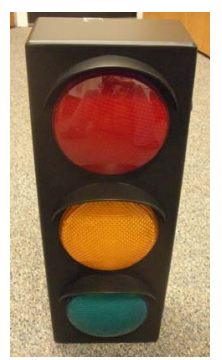

(a) Traffic light

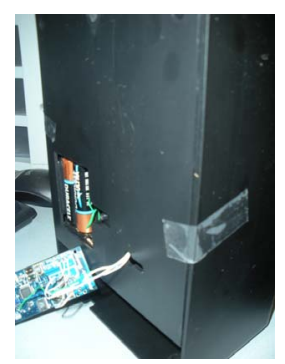

(b) Control mote
Fig. 1. (a) The off-the-shelf traffic light used in the LEVEL-TL implementation and (b) a picture of the control mote interface with the traffic light.

being inside two standard deviations of the baseline acoustic level mean. We assumed that samples were independent. The formula used to calculate LEVEL's $F N$ rate for a window is:

$$
P_{F N}(-\mid+)=P_{F N_{x}}(-\mid+)^{k}
$$

where $P_{F N_{x}}(-\mid+)$ is the $F N$ rate for a given sample $x$ and $k$ is the number of samples in a window. Thus, over 5 seconds, the probability of not detecting the teacher talking, when the teacher is talking, is around 0.00363 . This rate is acceptable.

We examined the false-positive $(F P)$ rate, or the probability of determining the teacher is talking, when the teacher is not talking. Each baseline acoustic level sample had a probability of approximately 0.0234 of being outside two standard deviations of the baseline acoustic level mean. The formula used to calculate LEVEL's $F P$ rate for a window is:

$$
P_{F P}(+\mid-)=1-\left(1-P_{F P_{x}}(+\mid-)\right)^{k}
$$

where $P_{F P_{x}}(+\mid-)$ is the $F P$ rate for a given sample $x$. Thus, over a period of 5 seconds, the probability of detecting the teacher talking, when the teacher is not talking, is around 0.11 . Thus, in one out of nine times, students will have an additional 5 second delay before they are signaled to raise their hands. This FP rate is acceptable for LEVEL's purposes.

\section{B. LEVEL-TL}

LEVEL-TL implements two motes: a teacher mote and a control mote. The teacher mote samples the acoustic sensor and classifies the sampled acoustic value as a normal value or as an anomalous value. Upon detecting an anomalous value (i.e., the teacher is speaking) the LEVEL-TL teacher mote broadcasts a 'red' packet. If a value is classified as normal, a speech timer is started in the teacher mote. This timer mitigates pauses in human speech. If a sampled acoustic value is sampled and classified as anomalous while the speech timer is running, the speech timer is canceled and a 'red' packet is sent to the control mote. The teacher mote monitors acoustic samples while the speech timer is running. If all samples are classified as normal, a 'green' packet is sent from the teacher mote to the control mote when the speech timer expires.

The control mote controls an off-the-shelf traffic light that signals to the students when it is acceptable to raise their hands. Figure 1 depicts the Lava Lite ${ }^{\mathrm{TM}}$ [11] traffic light used and how the control mote is wired to the traffic light.

The control mote receives either 'red' or 'green' directive packets from the teacher mote and changes the illuminated 


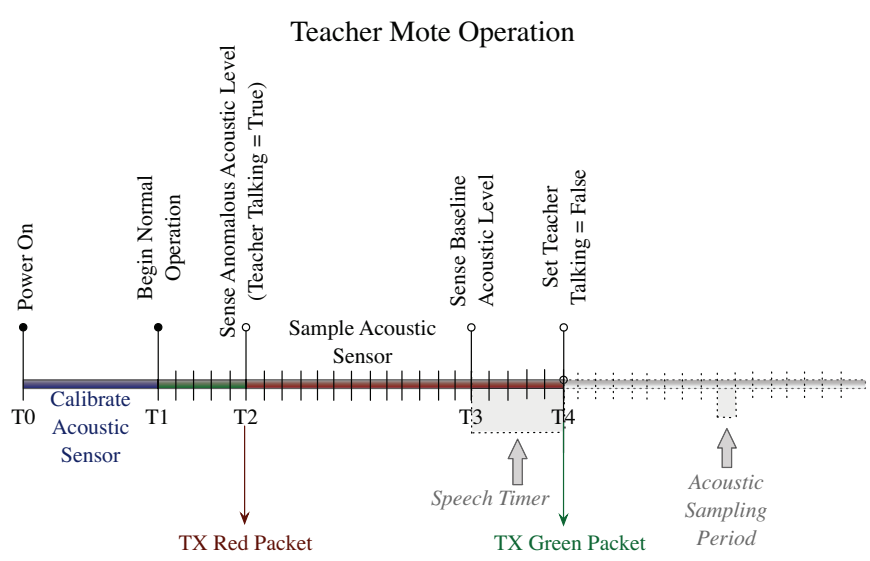

Fig. 2. teacher mote operation in LEVEL-TL and LEVEL-IS.

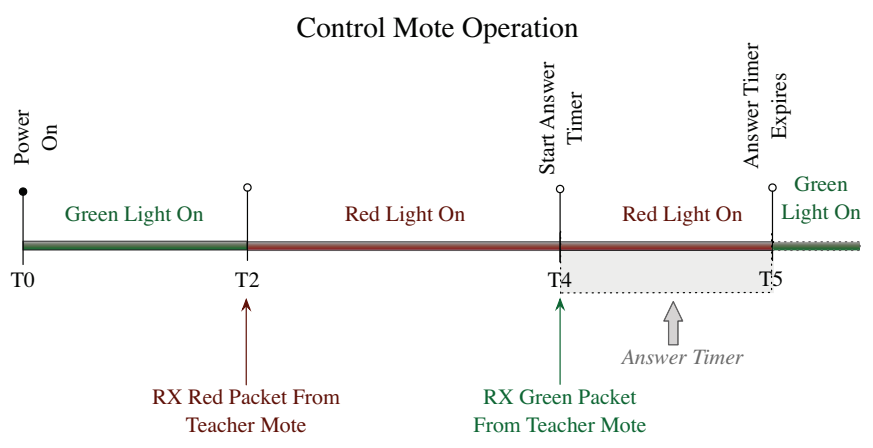

Fig. 3. control mote operation in LEVEL-TL.

traffic light due to these packets. When the control mote receives a 'red' packet from the teacher mote and the green LED is on, the control mote briefly illuminates the yellow LED, and then illuminates the red LED; the red LED informs students that they are not allowed to raise their hands to answer a question. Upon receiving a 'green' packet from the teacher mote, the control mote starts an answer timer.

The answer timer represents the amount of time for students to process and internalize the posed question before raising their hands. This timer gives students of all abilities a chance to determine an answer before classmates begin raising hands. In LEVEL-TL, the answer timer is constant. When the answer timer expires, the control mote illuminates the green LED on the traffic light, signaling to students that they may raise their hands to answer the question. Figures 2 and 3 depict teacher and control mote operation in $L E V E L-T L$, respectively.

\section{LEVEL-IS}

LEVEL-IS is the distributed version of LEVEL. LEVELIS uses $n+1$ motes: a teacher mote and $n$ additional student motes. Every mote in LEVEL-IS is equipped with an acoustic sensor. In LEVEL-IS, each student has a mote to allow for individualized answer delay timer feedback based on the student's previous participation (i.e., if a student has answered a question, the individual student's answer delay timer is increased). Student motes use the same anomaly detection algorithm as the teacher mote. LEVEL-IS teacher mote operation is similar to LEVEL-TL teacher mote operation; however, instead of communicating with a single control mote (as in LEVEL-TL), the LEVEL-IS teacher mote communicates with the student motes. Figures 2 and 4 depict teacher and student mote operation in LEVEL-IS, respectively.

1) Reliability: Communication in LEVEL-IS can be characterized as single broadcast delivery between teacher mote and student motes. Reliable communication between teacher mote and student motes is made possible through the use of ACKS. For simplicity, LEVEL-IS implements a customized version of B-MAC [12] with acknowledgments enabled.

In LEVEL-IS's customized version of B-MAC, each student mote waits a random time before transmitting a directive $A C K$ to avoid the ACK implosion problem documented in multicast protocols [13]. The teacher mote waits to receive directive ACKS during a directive ACK timeout period, which must be greater than the maximum random ACK back off time. If a directive $A C K$ is not received from student mote $\mathrm{B}$ within the directive ACK timeout period, then the teacher mote will transmit a unicast directive packet to student mote B. The unicast directive packet transmitted to student mote $\mathrm{B}$ includes a time offset equal to the elapsed time from when the broadcast directive packet was first sent. In this way, student mote B can adjust its answer timer to account for time elapsed between the initial broadcast of the directive packet and the actual reception of the directive packet. This adjustment allows each student mote's answer timer to expire at approximately the same time.

2) Fairness: LEVEL-IS implements a fairness algorithm on each student mote. LEVEL-IS's fairness algorithm is modeled after the TCP congestion control protocol. The fairness algorithm in LEVEL-IS employs a multiplicative increase on an individual student mote's answer timer when the student answers a question. An additive decrease is applied to the answer timer each time a student does not answer a question.

When a student mote's green LED is illuminated and the student mote's acoustic sensor samples anomalous values for longer than the sensitivity parameter, the algorithm employs a multiplicative increase on the student mote's answer timer. Figure 4 depicts details of the multiplicative increase mechanism performed on three student motes' answer timers. Every time the student does not answer a question, the student mote's answer timer is reduced through additive decrease.

\section{PARAmeters}

We empirically selected LEVEL's initial parameter values. Experiments determined that a sampled acoustic level outside of two standard deviations from the mean baseline ambient acoustic level signifies human speech. Other parameters determined empirically include the acoustic sampling period, the speech timer, and the answer timer. Parameters specific to LEVEL-IS determined empirically include the ACK delay timer, the multiplicative increase, and the additive decrease. A summary of LEVEL parameters are displayed in Table I.

\section{Classroom Deployment}

LEVEL-TL was deployed in a fifth grade classroom. During initial testing we discovered that the anomaly detection algorithm experienced excessive false negatives. We modified 


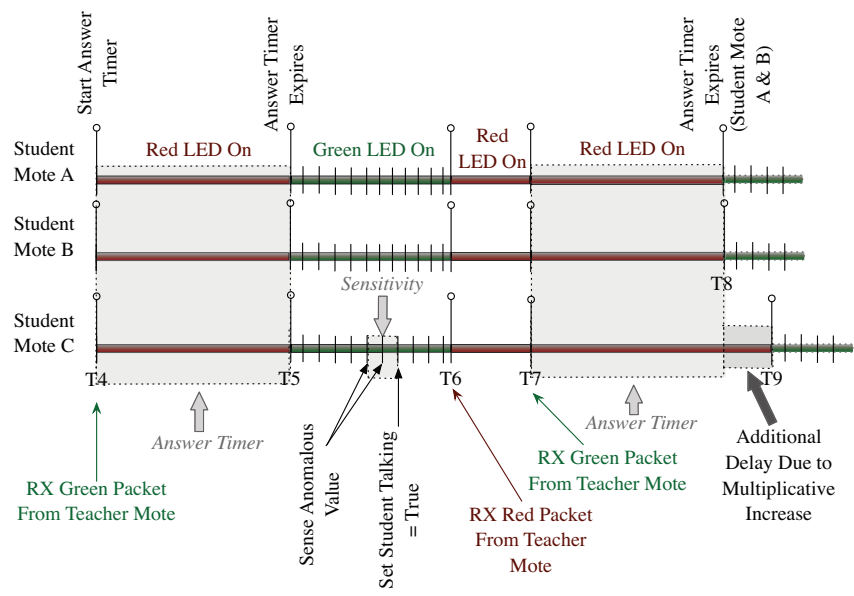

Fig. 4. Depiction of multiplicative increase in LEVEL-IS.

\begin{tabular}{|c|c|c|c|}
\hline Parameter & Description & $\begin{array}{c}\text { Empirical } \\
\text { Value }\end{array}$ & $\begin{array}{c}\text { Deployed } \\
\text { Value }\end{array}$ \\
\hline \hline \multicolumn{3}{|c|}{ Variables used in LEVEL-TL and $\boldsymbol{L E V E L - I S .}$} \\
\hline \hline $\begin{array}{c}\text { Calibration } \\
\text { Period }\end{array}$ & $\begin{array}{c}\text { Acoustic sensor } \\
\text { calibration period }\end{array}$ & $30 \mathrm{~s}$ & $15 \mathrm{~s}$ \\
\hline Sensitivity & $\begin{array}{c}\text { Anomaly detection } \\
\text { sensitivity parameter }\end{array}$ & 2 & 1.5 \\
\hline $\begin{array}{c}\text { Sampling } \\
\text { Period }\end{array}$ & $\begin{array}{c}\text { Time between } \\
\text { acoustic samples }\end{array}$ & $1 \mathrm{~s}$ & $0.1 \mathrm{~s}$ \\
\hline $\begin{array}{c}\text { Speech } \\
\text { Timer }\end{array}$ & $\begin{array}{c}\text { Delay before } \\
\text { send 'green' packet }\end{array}$ & $5 \mathrm{~s}$ & $5 \mathrm{~s}$ \\
\hline $\begin{array}{c}\text { Answer } \\
\text { Timer }\end{array}$ & $\begin{array}{c}\text { Student hand } \\
\text { raise delay }\end{array}$ & $15 \mathrm{~s}$ & $8 \mathrm{~s}$ \\
\hline \hline \multicolumn{4}{|c|}{ Variables specific to $\boldsymbol{L E V E L - I S .}$} \\
\hline \hline $\begin{array}{c}\text { ACK } \\
\text { Delay Timer }\end{array}$ & $\begin{array}{c}\text { Reliable } \\
\text { TX/RX delay }\end{array}$ & $5 \mathrm{~s}$ & N/A \\
\hline $\begin{array}{c}\text { Multiplicative } \\
\text { Increase }\end{array}$ & $\begin{array}{c}\text { Variable } m \text { in } \\
\text { fairness algorithm }\end{array}$ & 1.10 & N/A \\
\hline $\begin{array}{c}\text { Additive } \\
\text { Decrease }\end{array}$ & $\begin{array}{c}\text { Variable } a \text { in } \\
\text { fairness algorithm }\end{array}$ & $1 \mathrm{~s}$ & N/A \\
\hline
\end{tabular}

TABLE I

LEVEL PARAMETER DETAILS

the anomaly detection sensitivity parameter from the initial value of 2 to a value of 1.5 . This modification increased LEVEL's anomaly detection $F P$ rate and decreased its $F N$ rate. We were surprised that we needed this modification due to our analysis described in Section III-A. This modification may have been required because of acoustic differences in the classroom versus our testing area. After this modification, the qualitative experience of using LEVEL was improved for the $F N$ rate. Unfortunately, the $F P$ rate still remained qualitatively high.

To address the relatively high $F P$ rate, the acoustic sensor sampling period was decreased from 1 second to 0.1 second. Additionally, the algorithm was modified to require two adjacent anomalous values to indicate a state of 'talking'. Under the assumption of sample independence, this sampling rate adjustment reduces the $F P$ rate of LEVEL's anomaly detection algorithm. The formula used to calculate LEVEL's $F P$ rate for a single window in the classroom deployment is:

$$
P_{F P C}(+\mid-)=1-\left(1-P_{F P C_{x}}(+\mid-)^{2}\right)^{k}
$$

where $P_{F P C_{x}}(+\mid-)$ is the $F P$ rate for a given sample $x$ and $k$ is the number of samples in a window. If all parameter values are set to initial values (see Table I), the initial FP rate of 0.11 would be decreased to 0.027 . We were unable to quantitatively measure the $F P$ rate in the classroom. Qualitatively, the sampling period change improved the performance of the anomaly detection algorithm. Table I displays $L E V E L$ $T L$ parameter values deployed in the classroom.

\section{CONCLUSION}

This paper presents the design, implementation, and classroom deployment of a WSN to balance in-class participation. Listening to Everyone's Voice Enhances Learning (LEVEL) has two operating modes: centralized and distributed. Initial elementary school classroom deployment of the centralized version of LEVEL, LEVEL-TL, showed that the system successfully operates within a classroom setting. The classroom deployment improved understanding of the importance and impact of LEVEL's parameter values. LEVEL demonstrates that WSNs can be used to improve classroom participation.

The broader impacts of this work include introducing elementary school children to current technology used in computer science and expanding the exposure of elementary school teachers to novel teaching technologies. We hope to see future WSN systems integrated into classrooms.

\section{REFERENCES}

[1] T.-C. Liu, J.-K. Liang, H.-Y. Wang, T.-W. Chan, and L.-H. Wei, "Embedding EduClick in Classroom to Enhance Interaction," February 2005.

[2] J. N. Swift, "Two Effective Ways to Implement Wait Time. A Symposium on Wait Time." in Annual Meeting of the National Association for Research in Science Teaching, April 1985.

[3] J. N. Swift, C. T. Gooding, and P. R. Swift, "Using Research to Improve the Quality of Classroom Discussions," Research Matters - to the Science Teacher, no. 9601, October 1996.

[4] K. Tobin, "The Role of Wait Time in Higher Cognitive Level Learning," Review of Educational Research, vol. 57, no. 1, pp. 69-95, 1987.

[5] M. B. Rowe, "Wait-Time and Rewards as Instructional Variables: Their Influence on Language, Logic, and Fate Control," National Association for Research in Science Teaching, 1972.

[6] "Traffic Light Noise Meter Can Prevent Hearing Loss in Preschoolers," Med India Networking for Health, September 2009, http://www.medindia.net/news/Traffic-Light-Noise-Meter-can-PreventHearing-Loss-in-Preschoolers-58642-1.htm Accessed 4-6-2010.

[7] "Talk Light Inc." Online, www.talklight.com Accessed 4-6-2010.

[8] K. Stone, D. Hakkarinen, and T. camp, Listening to Everyone's Voice Enhances Learning (LEVEL), Technical Report, MCS-10-01, Colorado School of Mines, 2010, http://www.kerristone.net/resume/ technical-reports/LEVEL_MCS-10-01.pdf Accessed 7-21-2010.

[9] J. Polastre, R. Szewczyk, and D. Culler, "Telos: Enabling Ultra-low Power Wireless Research," in IPSN '05. Piscataway, NJ, USA: IEEE Press, 2005

[10] EasySen, "SBT80 Multi-Modality Sensor Board for TelosB Wireless Motes Data Sheet," 2008.

[11] "Lava Lite LLC," Online, http://www.lavalamp.com/ Accessed 4-9-2010.

[12] J. Polastre, J. Hill, and D. Culler, "Versatile Low Power Media Access for Wireless Sensor Networks," in SenSys '04. New York, NY, USA: ACM, 2004.

[13] S. Floyd, V. Jacobson, C.-G. Liu, S. McCanne, and L. Zhang, "A Reliable Multicast Framework for Light-weight Sessions and Application Level Framing," in IEEE/ACM Transactions on Networking, vol. 5, 1995. 